University of Wollongong

Research Online

Faculty of Informatics - Papers (Archive)

Faculty of Engineering and Information

Sciences

$14-5-2007$

\title{
A Study of Different Decoders for the New Rotation Spreading Matrix for Block Spread OFDM in UWB Channels
}

\author{
Ibrahim S. Raad \\ University of Wollongong, ibrahim@uow.edu.au \\ Xiaojing Huang \\ University of Wollongong, huang@uow.edu.au \\ Darryn Lowe \\ University of Wollongong, darrynl@uow.edu.au
}

Follow this and additional works at: https://ro.uow.edu.au/infopapers

Part of the Physical Sciences and Mathematics Commons

\section{Recommended Citation}

Raad, Ibrahim S.; Huang, Xiaojing; and Lowe, Darryn: A Study of Different Decoders for the New Rotation Spreading Matrix for Block Spread OFDM in UWB Channels 2007.

https://ro.uow.edu.au/infopapers/628

Research Online is the open access institutional repository for the University of Wollongong. For further information contact the UOW Library: research-pubs@uow.edu.au 


\title{
A Study of Different Decoders for the New Rotation Spreading Matrix for Block Spread OFDM in UWB Channels
}

\author{
Abstract \\ This paper presents a study into different decoders for the new Rotation Spreading Matrix proposed for \\ Block Spread OFDM (BSOFDM). It presents simulation results for the Maximum Likelihood (ML), \\ Minimum Mean Square Error (MMSE), Zero Forcing (ZF), Maximal Ratio Combining (MRC) and Equal Gain \\ Combining (EGC) decoders across the four IEEE defined Ultra-Wideband (UWB) channels CM1 to CM4. \\ While it is well known that the ML decoder should theoretically outperform all the other decoders, \\ practically due to its complexity it is not a viable option. It can be shown that MMSE, for BSOFDM with the \\ proposed new Rotation Spreading Matrix, has a good performance at low SNR and has been proven to be \\ a practical solution in wireless communications systems due to its simplicity and performance.

\section{Keywords} \\ BSOFDM, ML, MMSE, ZF, Block Spread-OFDM, UWB

\section{Disciplines} \\ Physical Sciences and Mathematics

\section{Publication Details} \\ This conference paper was originally published as Raad, IS, Huang, X, Lowe, D, A Study of Different \\ Decoders for the New Rotation Spreading Matrix for Block Spread OFDM in UWB Channels, IEEE \\ International Conference on Telecommunications and Malaysia International Conference on \\ Communications ICT-MICC 2007, 14-17 May, 372-376.
}




\title{
A Study of Different Decoders for the New Rotation Spreading Matrix for Block Spread OFDM in UWB Channels
}

\author{
Ibrahim S. Raad, Xiaojing Huang and Darryn Lowe \\ School of Electrical, Computer and Telecommunications Engineering \\ University of Wollongong, N.S.W Australia \\ ibrahim@uow.edu.au
}

\begin{abstract}
This paper presents a study into different decoders for the new Rotation Spreading Matrix proposed for Block Spread OFDM (BSOFDM). It presents simulation results for the Maximum Likelihood (ML), Minimum Mean Square Error (MMSE), Zero Forcing (ZF), Maximal Ratio Combining (MRC) and Equal Gain Combining (EGC) decoders across the four IEEE defined Ultra-Wideband (UWB) channels CM1 to CM4. While it is well known that the ML decoder should theoretically outperform all the other decoders, practically due to its complexity it is not a viable option. It can be shown that MMSE, for BSOFDM with the proposed new Rotation Spreading Matrix, has a good performance at low SNR and has been proven to be a practical solution in wireless communications systems due to its simplicity and performance. ${ }^{1}$
\end{abstract}

Key Words-BSOFDM, ML, MMSE, ZF, Block Spread$O F D M, U W B$

\section{Introduction}

Spreading matrices have been proposed to allow communications systems to improve their frequency diversity performance. Such systems as OFDM with the use of spreading matrices such as Hadamard, are now been called Block Spread OFDM (BSOFDM). The spreading matrices increase the correlation between the symbols, and as such improve the overall system performance in frequency selective channels. The new Rotation spreading matrix proposed in [1], is a method used to increase the correlation between the symbols through rotating the modulated symbols, and depending on the rotation angle, $\alpha$, a new and higher order modulation is used in the transmission of the system to increase the correlation between the transmitted symbols to improve the BER performance. This new Rotation spreading matrix, which has shown that it outperforms other spreading matrices, as with other existing ones like the Hadamard, are used in Block Spreading OFDM which are discussed in detail in [2], [3] and [5]. This is not the same as adaptive modulation as this does

\footnotetext{
${ }^{1}$ This research is sponsored by ARC DP 0558405
}

not retransmit the data when the new modulation scheme is achieved but rather has the same modulation scheme at the transmission. At the receiver, varies types of decoders can be used with BSOFDM. This paper studies five different decoders which include the Maximum Likelihood decoder (ML), which is theoretically the best, but due to complexity issues is not widely used. Other decoders that are studied include the Minimum Mean Square Error (MMSE) decoder, which has a good performance at low SNR, the Zero Forcing (ZF) decoder which is the simplest and has a similar performance to MMSE at high SNR, the Maximal Ratio Combining (MRC) decoder and the Equal Gain Combining (EGC). It can be shown for the proposed new Rotation spreading matrix, the MMSE at low SNR is a good option at the receiver but the $\mathrm{ZF}$ is also a viable option due to its simplicity and performance. This paper has the following sections. Section 2 briefly describes the Block Spread OFDM system used to test the varies decoders at the receiver. Section 3 discusses the new Rotation spreading matrix. Section 4 gives a brief outline of the different decoders that are to be used for this study. Section 5 presents the simulation results and finally the conclusion is given in Section 6.

\section{System Description}

Block Spread OFDM (BSOFDM) is when the full set of subcarriers are divided into smaller blocks and using spreading matrices to spread the data across these blocks so to achieve multipath diversity across each block at the receiver [2], [3] and [5]. The BSOFDM channel model is shown in Figure 1.

$$
y=C q+n
$$

The output of the receiver's FFT processor is given in Equation 1, where $y$ is the FFT output, $q \in A^{N}$ is the vector of transmitted symbols, each drawn from an alphabet $A, C$ is a diagonal matrix of complex normal fading coefficients, and $n$ is a zero mean complex normal random vector. Equalization of the received data is done through multiplication by $C^{-1}$ and then "quantized independently on each subcarrier to form the soft or hard decision $\hat{q}$ 


\begin{tabular}{|c|c|c|c|c|}
\hline & CM1 & CM2 & CM3 & CM4 \\
\hline Channel Model & LOS & NLOS & NLOS & NLOS \\
& $0-4 m$ & $0-4 m$ & $4-10 m$ & $10 m$ \\
Mean Ex. delay (ns) & 5 & 9.9 & 15.9 & 30.1 \\
RMS Delay (ns) & 5 & 8.0 & 15.0 & 25.0 \\
No. of multi-arrival & 12.5 & 15.3 & 24.9 & 41.2 \\
within 10dB of peak & & & & \\
\hline
\end{tabular}

Table 1. UWB channels defined by IEEE [7]

which may be further processed if the data bits are coded" [5]. There is no loss in performance when the detection is performed independently on each carrier due to the noise being independent and identically distributed with fading been diagonal [5].

The block spreading matrices are used to introduce dependence among the subcarriers. $N$ subcarriers are split into $\frac{N}{M}$ of blocks of size $\mathrm{M}$, where $M=2$ is used for this example. Then each of the blocks are multiplied by a $2 \times 2$ unitary matrix $U_{2}$. The length two output vectors are interleaved using general block interleaving to ensure the symbols are statistically independent so as to encounter independent fading channels. This will ensure in a dispersive frequency selective channel the data is statistically less likely to become corrupted and studies and simulations have shown this to be correct.

The transmitter's IFFT has the interleaved data passed through it and this data is sent across the frequency selective channel. For this experiment, the frequency selective channel used is the Ultra Wide Band channels, and this is where Ultra-wideband (UWB) systems occupy by definition - a signal spectrum of more than $500 \mathrm{MHz}$ or more than $20 \%$ with regards to their centre frequency. The application of such large bandwidths enables communication systems with unique novel properties, like highprecision indoor positioning.

Based on the Saleh-Valenzuela model for indoor multipath radio propagation channels, a set of statistically UWB channel models has been produced by the IEE E.802.15.3a task group [7]. Line of Sight (LOS) and Non-Line of Sight (NLOS) cases can be modelled using these standard channel models. The four different scenarios are summarized in table 1. Each are identified from $C M 1$ to $C M 4$. These channels are used in our study of different decoders for the new Rotation matrix as they represent a good example of frequency selective channels. The data is passed through an FFT processor at the receiver and deinterleaved before using block by block processing. The spreading matrices are generally used to increase the correlation between the transmitted symbols after the transmission has occurred. Unlike adaptive modulation schemes where depending on the system, a higher order modulation scheme is used to retransmit the data depending on the conditions presented, this scheme utilizes spreading matrices to increase the correlation between the symbols, rather than retransmitting. This is depicted in Figure 2. So say at the transmission the system modulates the data using QPSK modulation, with spreading matrices a higher order modulation is used to increase correla-

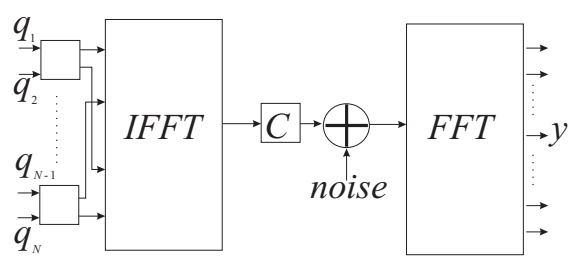
Figure 1. Block diagram representation of the BSOFDM channel for a block length of two [5].

tion and therefore overall system performance. There are a number of matrices available and well studied, this paper continues the study of the new Rotation spreading matrix presented in [1] and studies five different decoders at the receiver.

\section{New Rotation Spreading matrix}

In [1], a new spreading matrix known as the Rotation spreading matrix was proposed for Block Spread OFDM and the structure of this matrix is shown in Equation 2 and in Figure 2 it can be seen showing the new constellation achievable using the new spreading matrix with the angle $\alpha=\frac{\pi}{3}$ being used with the QPSK constellation scheme. This shows that the correlation between the blocks of size $M$ is increased and therefore in a frequency selective channel the performance in terms of BER is improved. It was shown in [4] that the angle $\frac{p i}{3}$ in Ultra Wide Band (UWB) channels had the best performance and can be seen in Figure 3.

$$
U=\left[\begin{array}{cc}
1 & \tan (\alpha) \\
\tan (\alpha) & -1
\end{array}\right]
$$

$\alpha$ is the angle used with the Rotation spreading matrix and as discussed in [1], the Rotation spreading matrix has been proven to outperform the Hadamard and the Rotate Hadamard and can be seen in Figure 4. It was noted that due to its flexibility in producing other matrices as well as unique configurations gave the Rotation matrix the advantage in frequency selective channels such as UWB.

\section{Decoders for BSOFDM}

If we assume there are $k$ subcarriers in an BSOFDM system and the channel to be described as $h(k)$, the received signal is described as $R(k)$ and the equalizer to be used to be described as $g(k)$, then for a one tap equalizer the expression can be presented as follows,

$$
C_{k}=g(k) \times R(k)
$$

For despreading using $M=2$ blocks, the following expression can be used to describe the process, 


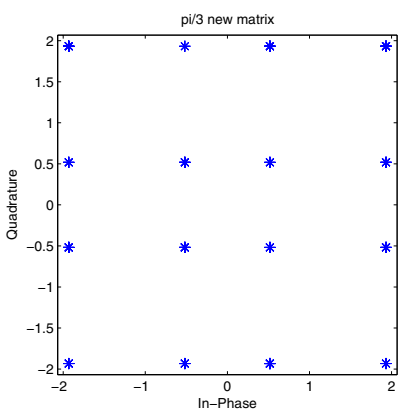

Figure 2. The new matrix for block spread OFDM with rotation $\frac{p i}{3}$.

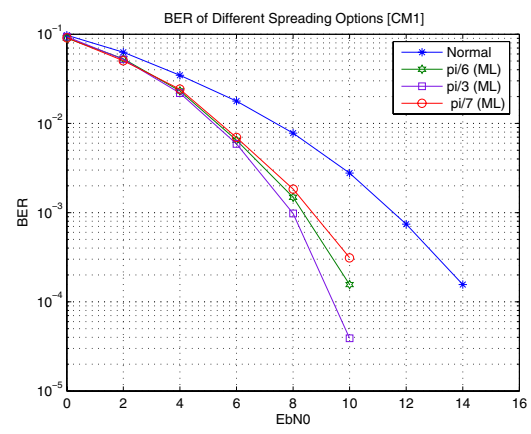

Figure 3. The new matrix with angles $\frac{p i}{6}, \frac{p i}{3}$ and $\frac{p i}{7}$.

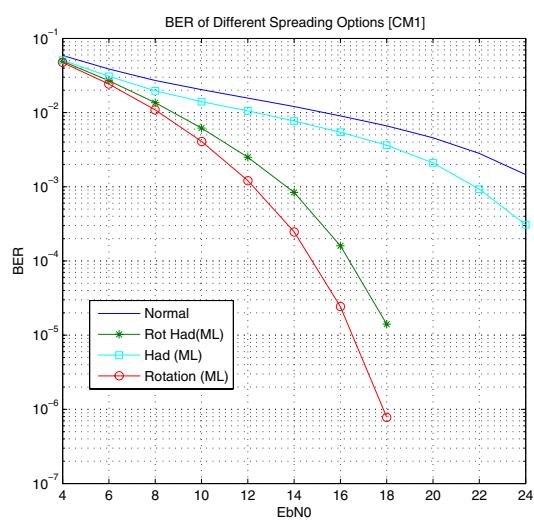

Figure 4. The new Rotation matrix shown outperforming Rotated Hadamard and Hadamard matrices in UWB $C M 1$.
Output $=U^{-1} \times\left[\begin{array}{c}g\left(k_{1}\right) \times R\left(k_{1}\right) \\ g\left(k_{2}\right) \times R\left(k_{2}\right)\end{array}\right]=\left[\begin{array}{c}\hat{a_{1}} \\ \hat{b_{2}}\end{array}\right]$

Where the $U^{-1}$ is the inverse of the unitary matrix used at the transmitter for spreading the blocks of $M$ size and $\hat{a_{1}}$ and $\hat{b_{2}}$ are the despread and decoded symbols at the receiver. Then the following decoders can be used in this study at the receiver.

\subsection{Maximum Likelihood (ML) Decoder}

The Maximum Likelihood Decoder (ML) is known to have the best performance of all the decoders presently available, as it calculates all possible combinations at the receiver before making a decision. This is also known to be the most complex and most system implementations avoid using this decoder due to this issue. The following is the mathematical expression used for the ML decoder [6], it can be shown that the maximum likelihood criterion for the receiver is,

$$
g(k)=\min _{z}\left|\frac{\hat{H} Z-X}{\sigma}\right|^{2}
$$

where $Z$ is the transmitted data and $\hat{H}$ is the channel estimate, $X$ is the received data with the following format,

$$
X=H Z+n
$$

where $n$ is the Gaussian noise with variance $\sigma^{2}$.

\subsection{Minimum Mean Square Error (MMSE) Decoding}

The MMSE is a useful alternative to that of the ML decoder described above as it can achieve good results in terms of Bit Error Rate (BER) at low Signal to Noise Ratio (SNR). This is less complex than the ML. The down side of this decoder is at high SNR, the performance is similar to that of the Zero Forcing (ZF) decoder but unlike the $\mathrm{ZF}$ decoder, as seen from Equation 9, the interferences $a$ and $b$ are not forced to zero and as such the noise is not amplified.

The MMSE can be described as follows,

$$
g_{k}=\frac{h_{k}^{*}}{\left|h_{k}\right|^{2}+\frac{1}{S N R_{c}}}
$$

Where $h_{k}$ is assumed to be the known channel. If at very high SNR, the equation above can be shown to be as follows,

$$
g_{k}=\frac{1}{h_{k}}
$$

which says that it is a ZF decoder. This can be seen in the results at high SNR. So the MMSE is a good solution for BSOFDM at low SNR because it allows a good 
compromise between noise and the ISI minimization. It is robust (avoids problems with channel zeros) and widely used in practice.

\subsection{Zero Forcing (ZF) decoding}

The Least Square (LS) or Zero forcing (ZF) decoder is the simplest method used for decoding. The complexity is also the least. It is described in Equation 8 and as discussed earlier at high SNR it is the same as that of the MMSE. With the ZF decoder, the interference is forced to zero, or assumed to be zero, which in turn amplifies the noise and can be seen from the following mathematical expression for BSOFDM if the first received symbol is expressed as $\hat{a}$ and the second is expressed as $\hat{b}$,

$$
\begin{aligned}
& \hat{a}=x a+y b+n \\
& \hat{b}=x b+y a+n
\end{aligned}
$$

The first equation shows that for the received symbol $a$, the interference of received symbol $b$ is also available. So if one is to use the zero forcing of Equation 8, then the noise $n$ is amplified.

\subsection{Maximal Ratio Combining (MRC) decoding}

The MRC is a decoder which is described as follows,

$$
g_{k}=h_{k}^{*}
$$

After the channel estimation has taken place, the conjugate of the estimated channel is calculated. This has the worst performance for BSOFDM between all the decoders discussed in this paper and can be seen in the results section. This is due to its inability to compensate for the spreading and despreading which takes place in BSOFDM.

\subsection{Equal Gain Combining (EGC) de- coding}

The Equal Gain Combining decoder does not attempt to equalize the effect of the channel distortion in any way, but it is desirable for its simplicity. The EGC decoder can be expressed as follows,

$$
g_{k}=\frac{h_{k}^{*}}{\left|h_{k}\right|}
$$

This has a similar expression to the MMSE, but unlike the MMSE, regardless of the SNR, the same format remains. the results shows that this does not have the same performance as MMSE, but has a better performance than the MRC.

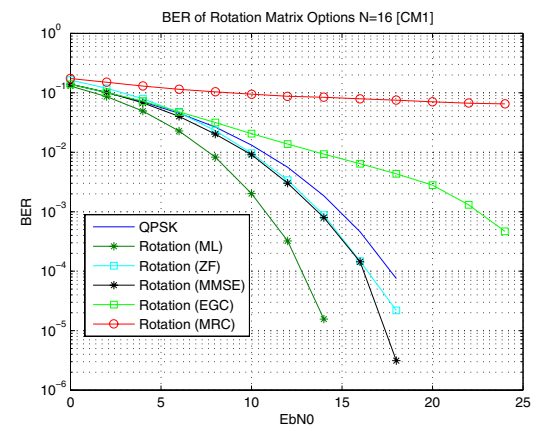

Figure 5. BER between MRC, EGC, MMSE, ZF and ML decoders Rotation spreading matrix N=16 for BSOFDM in UWB CM1.

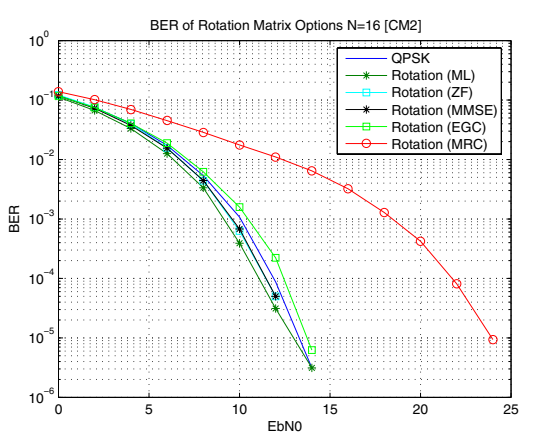

Figure 6. BER between MRC, EGC, MMSE, $\mathrm{ZF}$ and $\mathrm{ML}$ decoders Rotation spreading matrix $\mathrm{N}=16$ for BSOFDM in UWB CM2.

\section{Results}

For the simulation results the transmission is carried over the UWB channels discussed in Section 2 and that the cyclic prefix is of sufficient length (longer than the maximum path delay). We also assume that the channel is known at the receiver. The modulation scheme at the transmitter and receiver used is QPSK. The number of subcarriers used range from $N=16$ to $N=128$ with the number of packets simulated ranging from $a=10000$ to $a=100000$. Due to space limitation only $N=16$ subcarriers are shown from $C M 1$ to $C M 4$ UWB channels. As can be seen from Figures 5, 6, 7 and 8 the ML decoder performance the best in terms of BER as expected. This comes with the increased complexity as ML tries all different combinations at the receiver. The MMSE outperforms the ZF at smaller SNR and is less complex than the ML. But at high SNR, as can be seen from some of the results, the performance is the same as the ZF decoder. These same figures also show that the MRC decoder performs the worst out of the five decoders studied. The EGC decoder, while outperforming the MRC decoder, does not show any improvement over the nor- 


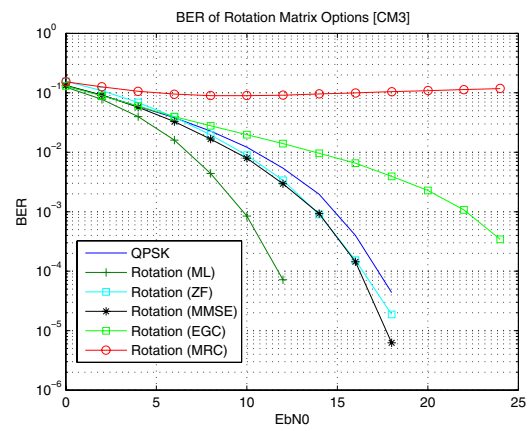

Figure 7. BER between MRC, EGC, MMSE, ZF and ML decoders Rotation spreading matrix $\mathrm{N}=16$ for BSOFDM in UWB CM3.

mal QPSK modulation although block spreading is used. So the advantage that the Rotation spreading matrix offers across UWB channels is not shown when using the MRC and the EGC decoding.

\section{Conclusion}

In conclusion this paper presented a study for different decoders that can be used with the new Rotation spreading matrix for BSOFDM system over the four channel models for UWB proposed for the IEEE.802.15.3a task group. The ML showed that it outperforms all the other decoders, as expected, due to it taking all possible combinations at the receiver and calculating the required constellation points. This comes at the expense of increased complexity as the size of the $\mathrm{N}$ subcarriers increased. The MMSE showed good performance at low SNR and was less complex than the ML. This has a good compromise between noise and ISI minimization and is widely used in practice due to its robustness. The disadvantage of such a decoder is at high SNR, as shown in Section 4.2, it has the same performance as the $\mathrm{ZF}$. Out of all the practical and useful decoders the ZF is the least complex and at high SNR has the same performance as the MMSE. For BSOFDM, in frequency selective channels such as the UWB, it continues to display the advantage of frequency diversity that BSOFDM shows.

The EGC decoder has a similar structure to the MMSE but does not take into account the SNR. This means, as shown by the results above, that any advantage offered by the frequency diversity of BSOFDM is lost and simply using a normal QPSK modulation rather than spreading with the Rotation matrix will yield a better result. Finally the MRC performs the worst out of the decoder proposed and the results show no improvement.

It can be concluded that for the new Rotation matrix for BSOFDM in frequency selective channels, such as UWB, that the MMSE be used as it offers the most practical solution in terms of complexity and performance. Today there are a number of alternative methods used with the MMSE

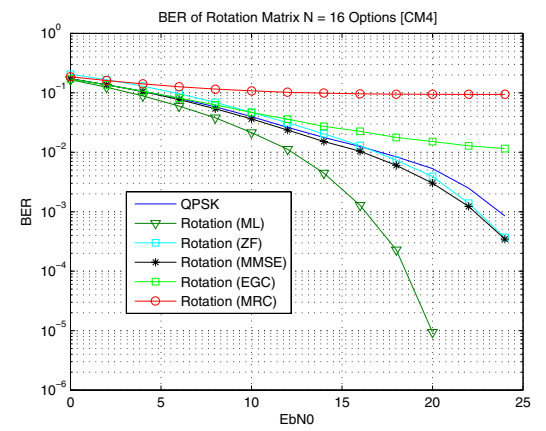

Figure 8. BER between MRC, EGC, MMSE, ZF and ML decoders Rotation spreading matrix N=16 for BSOFDM in UWB CM4.

to further help improve the performance while maintaining the robustness and the complex less structure.

\section{References}

[1] Ibrahim Raad, Xiaojing Huang and Raad Raad, "A New Spreading Matrix for Block Spread OFDM" 10th IEEE International Conference on Communication Systems 2006 (IEEE ICCS'06), Singapore, 31 October - 3 November 2006.

[2] Michael L. McCloud, "Coded block OFDM for the frequency selective fading channel", Signals, Systems and Computers, Conference Record of the Thirty-Eighth Asilomar Conference on, volume 1, 2004, 329-333, November.

[3] Michael L. McCloud, "Analysis and Design of Short Block OFDM Spreading Matrices for Use on Multipath Fading Channels", Communications, IEEE Transactions on, volume 53, 4, 2005, 656-66, April.

[4] Ibrahim Raad, Xiaojing Huang and Darryn Lowe , "A Study of different angles for the New Spread Matrix for BSOFDM in UWB channels" The Third International Conference on Wireless and Mobile Communications ICWMC 2007, March 4-9, 2007 Guadeloupe, French Caribbean.

[5] Michael L. McCloud, "Optimal Binary Spreading for Block OFDM on Multipath Fading Channels", WCNC / IEEE Communications Society, volume 2, 2004, 965-970, March.

[6] J. G. Proakis, Digital Communications, 4th ed. McGraw-Hill, 2000.

[7] Darryn W. Lowe, "Real - time FPGA Realization of an UWB transceiver physical layer" Thesis for Masters of Engineering Research at the University of Wollongong, The School of Electrical, Computer and Telecommunications Engineering, 2005. 